OPEN ACCESS

Edited by:

Debasish Bandyopadhyay,

The University of Texas-Pan American,

USA

Reviewed by:

Simone Brogi,

University of Siena, Italy

Joydip Das,

Indian Institute of Technology, India

*Correspondence:

Pedro J. Ballester

pedro.ballester@inserm.fr

Specialty section: This article was submitted to Medicinal and Pharmaceutical

Chemistry,

a section of the journal

Frontiers in Chemistry

Received: 24 February 2016

Accepted: 24 March 2016

Published: 14 April 2016

Citation:

Peón A, Dang CC and Ballester PJ (2016) How Reliable Are Ligand-Centric Methods for Target

Fishing? Front. Chem. 4:15.

doi: 10.3389/fchem.2016.00015

\section{How Reliable Are Ligand-Centric Methods for Target Fishing?}

\author{
Antonio Peón, Cuong C. Dang and Pedro J. Ballester* \\ Cancer Research Center of Marseille (Institut National de la Santé et de la Recherche Médicale U1068, Institut \\ Paoli-Calmettes, Aix-Marseille Université, Centre National de la Recherche Scientifique UMR7258), Marseille, France
}

Computational methods for Target Fishing (TF), also known as Target Prediction or Polypharmacology Prediction, can be used to discover new targets for small-molecule drugs. This may result in repositioning the drug in a new indication or improving our current understanding of its efficacy and side effects. While there is a substantial body of research on TF methods, there is still a need to improve their validation, which is often limited to a small part of the available targets and not easily interpretable by the user. Here we discuss how target-centric TF methods are inherently limited by the number of targets that can possibly predict (this number is by construction much larger in ligand-centric techniques). We also propose a new benchmark to validate TF methods, which is particularly suited to analyse how predictive performance varies with the query molecule. On average over approved drugs, we estimate that only five predicted targets will have to be tested to find two true targets with submicromolar potency (a strong variability in performance is however observed). In addition, we find that an approved drug has currently an average of eight known targets, which reinforces the notion that polypharmacology is a common and strong event. Furthermore, with the assistance of a control group of randomly-selected molecules, we show that the targets of approved drugs are generally harder to predict. The benchmark and a simple target prediction method to use as a performance baseline are available at http://ballester.marseille.inserm.fr/TF-benchmark.tar.gz.

Keywords: target prediction, virtual screening, polypharmacology prediction, drug repositioning

\section{INTRODUCTION}

Target Fishing (TF) (Cereto-Massagué et al., 2015; Lavecchia and Cerchia, 2015), also known as Target Prediction or Polypharmacology Prediction, consists in predicting the macromolecular targets of a query molecule. This problem is the reverse of Virtual Screening (VS) (Schneider, 2010; Sukumar and Das, 2011), where the goal is to predict the ligands of a query target. Computational methods for TF are of great interest, as identifying previously unknown targets of a molecule is the basis of a number of important drug design and chemical biology applications (Ursu and Waldmann, 2015). Indeed, discovering a new target for a drug could lead to its reposition in a new indication as well as an enhanced understanding of its efficacy and side-effects (Huang et al., 2014). Furthermore, these tools can be used for target deconvolution of phenotypic screening hits (Lee and Bogyo, 2013), which is a prerequisite to gain mechanistic understanding of phenotypic activity and helpful for drug development. This two-stage process, phenotypic screening followed by 
target deconvolution, constitutes an attractive alternative strategy for the discovery of molecularly targeted therapies.

The fast growth of freely-available bioactivity resources, e.g., PubChem (Cheng et al., 2014) or ChEMBL (Bento et al., 2014), has sparked a new generation of powerful data-driven methods for TF. This growth is exemplified by the ChEMBL database, which in a few years has assembled and fully curated chemical structures and bioactivities from more than 50,000 scientific publications (Bento et al., 2014). Moreover, this database is periodically updated and will eventually incorporate a flood of new data that is being extracted from the patent literature (Papadatos et al., 2016). As previously discussed (CeretoMassagué et al., 2015), TF methods have been categorized into those based on molecular similarity (Liu et al., 2014), machine learning (van Laarhoven et al., 2011), protein structure analysis (Gao et al., 2008), and bioactivity spectra analysis (Füllbeck et al., 2009; Holbeck et al., 2010). Some of these methods have been made available as web servers (Wang et al., 2013; Gfeller et al., 2014).

Here we propose a new classification of TF methods into two broad categories: target-centric and ligand-centric. Target-centric methods are defined as those building a predictive model for each considered target. Each of these models is thereafter used to predict whether the query molecule has activity against the corresponding target (other names for the query molecule are common, such as test compound, test molecule, or test ligand). Thus, this panel of models provides a set of predicted targets for any query molecule. Many target-centric TF methods are based on multi-target Quantitative Structure-Activity Relationship (QSAR) models (Zanni et al., 2014; Speck-Planche and Cordeiro, 2015). The model is typically trained on large sets of active and inactive target-ligand instances derived from a database of target-annotated molecules (the training set). These models have employed various regression or classification techniques, such as Kernel Classifiers (van Laarhoven et al., 2011), Winnow (Nigsch et al., 2008), Ranking Perceptron (Yu et al., 2012), Random Forest (Yu et al., 2012), or Naïve Bayes Classifier (Koutsoukas et al., 2013). Instead of supervised learning, other target-centric techniques are based on unsupervised learning such as the Similarity Ensemble Approach (SEA) (Keiser et al., 2007, 2009). SEA constructs a model for each target estimating how likely is the query molecule to belong to the set of cognate ligands of the target based on an underlying molecular similarity metric. In addition, there are target-centric methods that can estimate whether the query molecule binds to a structural model of the target (Schomburg and Rarey, 2014). These methods are in principle able to interrogate targets without known ligands, although their success largely depends on the accuracy of the employed scoring function (Ain et al., 2015).

On the other hand, ligand-centric methods are those based on the similarity of the query molecule to a very large set of target-annotated molecules. This similarity can be in terms of 2D chemical structure (Nettles et al., 2006) using circular fingerprints (Rogers and Hahn, 2010), 3D molecular properties (CortésCabrera et al., 2013) using Ultrafast Shape Recognition variants (Ballester and Richards, 2007; Armstrong et al., 2010; Ballester, 2011) or NCI-60 bioactivity spectra (Holbeck et al., 2010) using cellular fingerprints (Füllbeck et al., 2009). Note that there are similarity-based methods that are not ligand-centric. This is the case of TAMOSIC (Wang et al., 2013), which learns the optimal similarity cutoff for each target with at least 30 ligands.

An important advantage of ligand-centric methods over target-centric methods has been so far overlooked. Whereas, ligand-centric methods can interrogate any target that has at least one known ligand, target-centric models can only evaluate the typically much smaller set of targets for which a model can be built. For instance, TarFisDock (Gao et al., 2008) predictions are limited to 1100 targets with available crystal structure and known binding site, whereas SEA (Keiser et al., 2007, 2009) only evaluates targets with at least five known ligands. This means that target-centric methods are by construction blind to up to thousands of targets considered by ligand-centric techniques, but this is not obvious as target-centric performance is only evaluated on qualifying targets. Target-centric and ligand-centric methods are nevertheless complementary. Indeed, in cases where the targets of interest are known to have many ligands, more accurate target-centric models could be possible and thus these tools are likely to be more suitable. By contrast, in cases where evaluating as many targets as possible is preferable, ligand-centric tools would be more appealing, as these provide a much wider coverage of the proteome.

Unfortunately, it is unclear how well ligand-centric methods work in practice due to the limitations of existing benchmarks. Some validations have been restricted to a few tens of ligandrich targets using benchmarks borrowed from VS (AbdulHameed et al., 2012) and thus tell us very little about how well the methods will perform on the many remaining targets. Furthermore, some performance measures, such as the Receiver Operating Characteristic (ROC), Area Under Curve (AUC), do not precisely measure TF performance. For example, how many true targets of a query molecule one is likely to find in practice using a method that has obtained an average ROC AUC of 0.7 over 40 targets? On the other hand, TF is often posed as a multi-category classification problem, which formulates a binary classification problem per target and thus the variation of predictive performance across query molecules has not been analyzed in these studies. Importantly, these benchmarks exclude many possible targets of the analyzed molecules because the corresponding target-centric models could not be trained on the excluded targets. As a result of these limitations, current benchmarks offer little guidance on pragmatic questions such as how many predicted targets have to be tested on average to find a true target, how many known targets are typically missed or how such performance varies with the query molecule.

In this study, we propose a new benchmark to validate TF methods, which naturally lends itself to answer such questions. This is based on formulating a binary classification problem for each query molecule. From this new perspective, we provide a lower-bound for the current performance of ligand-centric methods representing the minimum that can be expected nowadays from them. As a byproduct, our analysis provides an update for the degree of polypharmacology observed in approved drugs. The rest of the paper is organized as follows. Section Experimental Setup describes the experimental setup, including 
data selection, data partitions, TF method and performance metrics. Section Results and Discussion discusses the results. Section Conclusions presents the conclusions.

\section{EXPERIMENTAL SETUP}

This section describes the setup of all the numerical experiments carried out in this study. This setup is composed of the following elements: data selection, data partitions, TF method, and measures of predictive performance. All molecular data processing is done with SQL queries from Python 2.7.9 on a local copy of the ChEMBL database running PostgreSQL 9.4.3, with molecular similarity searches using in addition the RDKit PostgreSQL cartridge (2015.03.1 release).

\section{Data Selection}

The first step is constructing datasets from the ChEMBL database. We started by downloading release 20 as a PostgreSQL dump (ChEMBL_20 release $^{1}$ ), which contains data for 10,774 targets, 1,456,020 molecules with disclosed chemical structure and 13,520,737 bioactivities.

Single-protein was the most common target type (6018 of the 10,774 targets). In order to provide the most specific target prediction, we restricted to single-protein targets, which incidentally constitutes the largest molecular target type in the database (the "protein complex," "protein family," and "nucleicacid" types only have 261, 217, and 29 targets, respectively). The remaining general constraints were requiring the maximum confidence_score $=9$ (i.e., direct single-protein target assigned by the data curator), activities.published_relation = '=', assay_type $=$ ' $\mathrm{B}$ ' and standard_units = ' $\mathrm{nM}$ '. As a result of this process, 888,354 molecules were found to be associated to the 6018 single-protein targets through 4,871,527 bioactivities.

Further requirements are commonly imposed for the measured bioactivity of a ligand against a target to be counted as a known target for that ligand. First, the bioactivity measurement must be of relatively high quality, activities.standard_type IN ('EC50,'Ki', Kd,'IC50'), which discards percentages of inhibition among other lower-quality measurements. Second, only complexes with a sufficiently potent bioactivity are retained (common activity thresholds are 1 and $10 \mu \mathrm{M}$ meaning that a ligand hitting any target with an activity higher than $10 \mu \mathrm{M}$ will not be considered to be a target in neither of these two scenarios). Third, only targets with at least $\mathrm{n}$ qualifying ligands are considered. For many target-centric methods, a sufficiently high number of ligands is needed to build a model for the target, e.g., those methods based on similarity-ensemble approaches $(n=5)$ (Keiser et al., 2009) or multi-target QSAR $(n=20)$ (Koutsoukas et al., 2013). In this study, we analyse ligand-centric methods, which can evaluate any target with at least a known ligand (i.e., $n=1$ ) and hence result in a much broader search for targets (3035 molecular targets with $10 \mu \mathrm{M})$. An analysis of the target coverage of TF methods is carried out in Section

${ }^{1}$ ChEMBL_20 release Available at: ftp://ftp.ebi.ac.uk/pub/databases/chembl/ ChEMBLdb/releases/chembl_20/(Accessed March 4, 2015)
How Many Targets Are Being Neglected by Target-Centric TF Techniques.

\section{Data Partitions}

Next, we partition each of the two $n=1$ datasets as follows. First, we identify the subset of approved drugs. Second, we search for all those approved drugs in the ChEMBL database meeting the criteria, with a suitable chemical structure available and hitting any of the targets introduced in the previous section. These are the two approved-drugs sets of query molecules shown in Table 1. Third, we pick at random two further sets of molecules of the same size, which we called random-molecules sets. This will serve as a control group to investigate how target predictions for marketed drugs differ from those made for other types of molecules. The rest of ligands forms the set of database molecules, which is the same for both sets of query molecules but different between thresholds. Table 1 shows the four non-overlapping data partitions A-D (no query molecule is included as database molecule too).

\section{A Simple TF Method to Estimate a Lower-Bound for Performance}

For our analysis, we selected a simple two-dimensional chemical similarity search (Willett, 2014) in order to obtain a lowerbound for the performance of ligand-centric TF methods. This goal requires selecting a simple method, rather than an optimal method which would be unlikely to provide such lower-bound. Consequently, we selected the dice score on MACCS fingerprints ad hoc, although there are of course other valid choices too. We started by generating MACCS fingerprints (Durant et al., 2002) for all query and database molecules in Table 1. Each fingerprint encodes the presence or absence of 166 predetermined chemical groups in the molecule as a binary string of the same size. These were generated using the RDKit (Lamdrum).

As usual, fingerprints could not be generated for a few unusual molecules and consequently queries could not be performed for these. This is the case of Gramidicin (CHEMBL1201469), which is actually not a molecule but a mixture of three antibiotic compounds. Other examples are some organometallic compounds such as the anti-rheumatic agent Auranofin (CHEMBL1366). Table 1 compiles all selected molecules for which MACCS fingerprints could be generated.

Using their MACCS fingerprints, the Dice score was used to measure the similarity between a query molecule and all the database molecules. The Dice score is defined as:

$$
\text { Dice }=2 \mathrm{c} /(\mathrm{a}+\mathrm{b})
$$

\section{TABLE 1 | Dataset size depending on modeling constraints.}

\begin{tabular}{llcc}
\hline ID & Dataset & $\begin{array}{c}\text { Number of } \\
\text { query-molecules database-molecules }\end{array}$ & $\begin{array}{c}\text { Number of } \\
\end{array}$ \\
\hline A & Approved-drugs_Thres 10 $\mu \mathrm{M}$ & 745 & 183,282 \\
B & Random-molecules_Thres 10 $\mu \mathrm{M}$ & 745 & 183,282 \\
C & Approved-drugs_Thres 1 $\mu \mathrm{M}$ & 617 & 147,027 \\
D & Random-molecules_Thres 1 $\mu \mathrm{M}$ & 617 & 147,027
\end{tabular}


where $\mathrm{a}$ is the number of on bits in molecule $\mathrm{A}, \mathrm{b}$ is number of on bits in molecule $\mathrm{B}$, while $\mathrm{c}$ is the number of bits that are on at the same positions in both molecules. For each query, the top $k$ hits can be identified from the corresponding ranking of database molecules (these are the $k$ database molecules with the most similar chemical structure to that of the query molecule). We consider here $k=1,5,10$, and 15 to investigate the dependence of the method with its only control parameter $k$.

Finally, the known targets for the $k$ hits are retrieved from the ChEMBL database and returned as predicted targets for the considered query molecule. Thus, a set of predicted targets is obtained for each combination of query molecule and $k$ value. Note that a known target is not just any target annotated in the ChEMBL database, but one complying with the requirements set in Section Data Selection for each of the four cases in Table 1.

\section{Measuring Predictive Performance}

Each performed query can be posed as a separate classification problem. For validation purposes, the known targets of the query molecule are taken as a ground truth. Thus, we assume that the known targets are all the qualifying targets of the molecule, whereas the rest of considered targets are non-targets for that molecule. However, as the query molecule has only been tested against $<0.1 \%$ of the ChEMBL targets on average, it is expected that many unconfirmed targets, especially those coming from molecules similar to the query molecule, would be actually targets if only these could be comprehensively tested. As a result, any empirically untested target-ligand association that is predicted to be a true association will have to be rejected as false, despite an unknown part of these being actually true targets of the molecule. We must therefore keep in mind that this retrospective validation represents a lower-bound for performance in this sense as well.

Table 2 shows the confusion matrix arising from assessing target predictions against experimental evidence for each query molecule. After the assessment, each target prediction can be classed in one of four categories: TP for True Positive (the predicted target is a known target); TN for True Negative (the target was not predicted but anyway is not known to be a target); FP for False Positive (the predicted target is not known to be a target, i.e., a false discovery or Type I error); and FN for False Negative (the target was not predicted and it is actually a target, i.e., missed discovery or Type II error).

From these quantities, we will calculate four performance measures per query molecule. Accuracy is the proportion of correct target predictions:

$$
\text { Accuracy }=\frac{T P+T N}{T P+F P+T N+F N}
$$

TABLE 2 | Confusion matrix arising from assessing target predictions against experimental evidence for each query molecule.

\begin{tabular}{lll}
\hline Target & Predicted & Non-predicted \\
\hline Yes (experimentally tested) & TP & FN \\
No (not tested/tested) & FP & TN
\end{tabular}

Precision is the proportion of new targets that would be obtained after experimentally validating the predictions of the method:

$$
\begin{aligned}
\text { Precision }= & \frac{\text { Number of known targets correctly predicted }}{\text { Number of predicted targets }} \\
& =\frac{T P}{T P+F P}
\end{aligned}
$$

Recall accounts for the proportion of true targets that the method has missed:

$$
\begin{aligned}
\text { Recall }= & \frac{\text { Number of known targets correctly predicted }}{\text { Number of known targets }} \\
& =\frac{T P}{T P+F N}
\end{aligned}
$$

The Matthews Correlation Coefficient (MCC) captures both types of error in a single metric, with higher values being better up to +1 (perfect classification):

$$
M C C=\frac{T P \times T N-F P \times F N}{\sqrt{(T P+F P)(T P+F N)(T N+F P)(T N+F N)}}
$$

Lastly, the Number of Predicted Targets (NPT) will be also reported to investigate how this varies with the method's control parameter $k$. The entire workflow is sketched in Figure 1.

\section{RESULTS AND DISCUSSION}

Four key questions along with two representative case studies are addressed in this section. The analysis is based on the performance obtained by the query molecules in the four datasets in Table 2, which will be summarized with boxplots of precision, recall, MCC and NPT.

\section{How Many Targets Are Being Neglected by Target-Centric TF Techniques?}

The first two rows of Table 3 show the number of targets considered by a ligand-centric TF method with two target definitions (i.e., activity thresholds of $1 \mu \mathrm{M}$ and $10 \mu \mathrm{M}$ ). The remaining rows show the number of targets considered by exemplary target-centric methods as a result of only considering targets with at least 5-40 ligands. To allow a fair comparison, we have calculated the number of targets using the same selection criteria on chembl20 data (Section Data Selection), except for the minimum number of ligands required by each method and the selected activity threshold.

For example, target-centric methods powered by models requiring at least 40 ligands per target and defining a target with an activity threshold of $10 \mu \mathrm{M}$ would be predicting whether the query molecule has activity against any of the 917 qualifying single-protein targets. In contrast, a ligand-centric method with the same activity threshold will be able to evaluate 2118 targets more, for which the first method is unable to provide any prediction by construction. Of course, the advantage of targetcentric over ligand-centric methods is that the former will tend to perform better on those targets with a high number of ligands, 


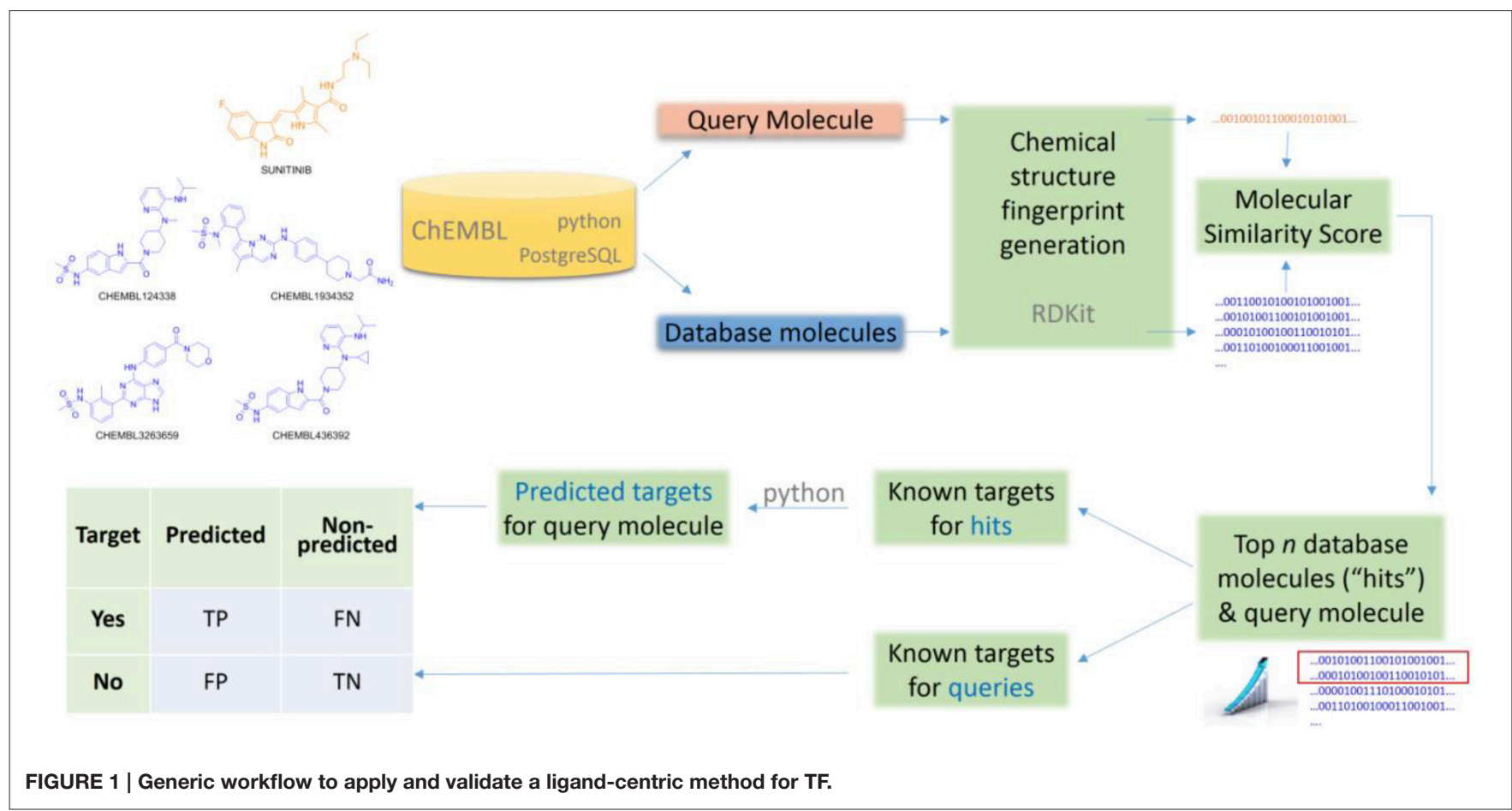

TABLE 3 | Numbers of considered targets and number of neglected targets depending on the data selection criteria of the employed benchmark.

\begin{tabular}{|c|c|c|c|c|c|}
\hline Dataset & $\begin{array}{l}\text { Only targets with } \\
\text { at least }\end{array}$ & $\begin{array}{c}\text { Only targets with } \\
\text { activity below }(\mu \mathrm{M})\end{array}$ & $\begin{array}{c}\text { Number of } \\
\text { targets in study }\end{array}$ & $\begin{array}{c}\text { Number of targets if } \\
\text { chembl20 }\end{array}$ & $\begin{array}{c}\text { Number of } \\
\text { targets neglected }\end{array}$ \\
\hline This study & 1 ligand & 1 & 2580 & 2580 & 0 \\
\hline This study & 1 ligand & 10 & 3035 & 3035 & 0 \\
\hline Keiser et al., 2007 & 5 ligands & 1 & 246 & 1788 & 792 \\
\hline Mugumbate et al., 2015 & 10 ligands & 10 & 1543 & 1804 & 1231 \\
\hline Koutsoukas et al., 2013 & 20 ligands & 10 & 894 & 1378 & 1657 \\
\hline Wang et al., 2013 & 30 ligands & 10 & 794 & 1104 & 1931 \\
\hline Martínez-Jiménez et al., 2013 & 40 ligands & 10 & 1258 & 917 & 2118 \\
\hline
\end{tabular}

which highlights the complementarity of both approaches. It would be interesting if the performance of target-centric methods was evaluated per target and analyzed against its number of cognate ligands, as it is currently unknown how reliable are their predictions on the many targets with only a few ligands above the minimum.

\section{How Many Targets Are Typically Hit by a Molecule?}

For each of the four cases in Table 2, Figure 2 shows boxplots summarizing the distribution of the number of known singleprotein targets (NKTs) across query molecules.

On the left, a substantial number of strong outliers are appreciated. These correspond to promiscuous query molecules such as sunitinib, which has 192 submicromolar targets (262 targets using the $10 \mu \mathrm{M}$ threshold). In contrast, there are also seemingly selective drugs like the antiretroviral agent Nelfinavir with only one known target below $1 \mu \mathrm{M}$ (HIV-1 protease; although there are also many non-molecular targets annotated in ChEMBL for this drug). On the right, we can appreciate that approved drugs currently have an average of eight known targets with potency better than $10 \mu \mathrm{M}$, although the median number is three targets. This new estimate is based on 745 drugs and their 1076 targets and it is two targets higher than previous estimates using less data (802 drugs and 480 targets) (Mestres et al., 2009). However, the boxplot's lower quartile value indicates that at least $25 \%$ of these drugs have just one known target and thus seem very selective. It is also noteworthy in Figure $\mathbf{2}$ that the number of annotated targets for the set of random molecules is smaller than that for approved drugs, with four targets on average instead of eight. This substantial difference is likely to be due to a much higher number of targets being tested during the process of developing a drug.

\section{How Many Predicted Targets Have to be Tested to Find a True Target?}

Table 4 presents average performance results for approved drugs (set A), with the TF method using four different $k$ values. As 

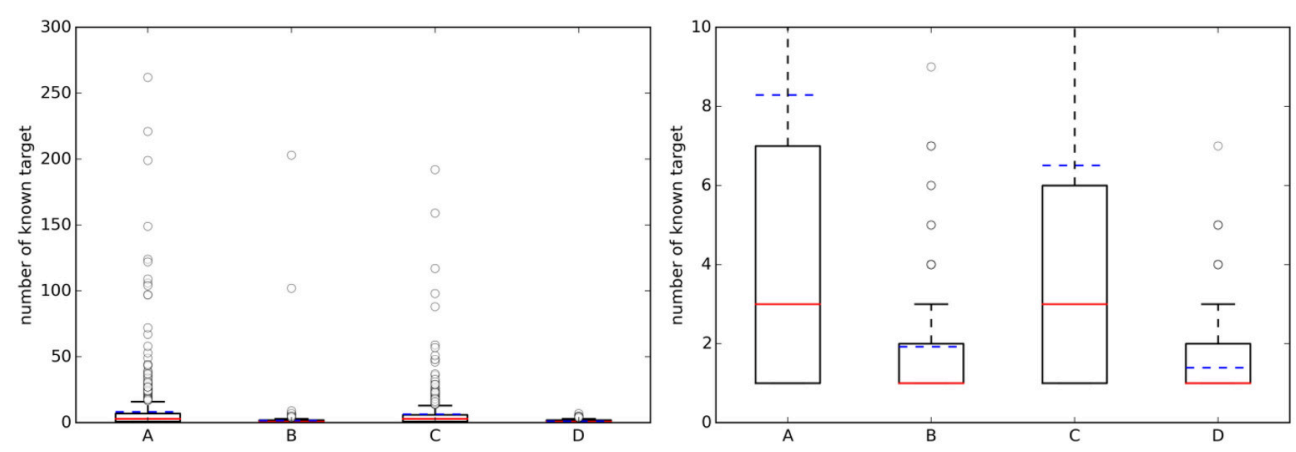

FIGURE 2 | (Left) Boxplots with the number of known targets (NKT) across query molecules. (Right) A zoom of the same boxplots on the right (the average number of known targets is marked with a dashed blue line, whereas the median is given by the continuous red line). A, Approved, $10 \mu \mathrm{M}$; $\mathrm{B}, \mathrm{Random}, 10 \mu \mathrm{M} ; \mathrm{C}, \mathrm{Approved}$, $1 \mu \mathrm{M} ; \mathrm{D}$, Random, $1 \mu \mathrm{M}$.

TABLE 4 | Average (av) performance of the TF method on query molecules from set $A=$ (Approved, $10 \mu \mathrm{M}$ ).

\begin{tabular}{lccccc}
\hline $\mathbf{k}$ & avNPT & avAccuracy & avPrecision & avRecall & avMCC \\
\hline 1 & 1.92 & 0.997 & 0.434 & 0.212 & 0.269 \\
5 & 5.04 & 0.997 & 0.352 & 0.341 & 0.303 \\
10 & 7.91 & 0.996 & 0.296 & 0.403 & 0.300 \\
15 & 10.32 & 0.995 & 0.257 & 0.437 & 0.289 \\
\hline
\end{tabular}

These molecules have an average of 8.3 known targets.

$k$ increases, Type I errors increase (lower precision) and Type II errors decrease (higher recall). In other words, as more top hits are used to provide predicted targets, fewer known targets are missed. However, this comes at the cost of having more false positives, as target inferences are made using increasingly less similar database molecules. Using the top 5 hits to predict targets (i.e., $k=5$ ) provides the best compromise between these conflictive objectives (i.e., the highest average MCC). This setting leads to 5.04 predicted targets on average over these query molecules (note that each top hit may have more than one known target, but collectively provide fewer targets because some of these are repeated in the set). Lastly, the very high average accuracy values are due to each classification problem being highly unbalanced and the method correctly discarding the vast majority of non-targets. Nevertheless, unlike precision and recall, accuracy is not suitable to measure Type I and II errors and hence is not helpful to address the investigated questions.

Figure 3 shows the distribution of the NPT across query molecules using $k=5$. By comparing it with Figure 2, it is observed that there are substantially more known targets than predicted targets for approved drugs using the top 5 hits for predictions (this is not the case for the sets of random molecules, where most query molecules have a higher number of predicted targets than of known targets).

Figure 4(left) summarizes the distribution of precision results across the query molecules. For approved drugs, the average precision is 0.35 in the $10 \mu \mathrm{M}$ case $(0.38$ in the $1 \mu \mathrm{M}$ case). That is, despite the simplicity of the method and thanks to the wealth of data on which it relies, only five predicted targets will have to be tested in order to find two true targets with potency better than 1 $\mu \mathrm{M}$. In all cases, there is strong performance variability across the query molecules, as it can be appreciated by the large interquartile range of each boxplot. For instance, in set $\mathrm{C}$, the predictions for the targets of 109 drugs are of the highest precision (precision $=1$ ), those for other 216 drugs not precise at all (precision $=0$ ) and those for the remaining 420 drugs have intermediate precision values (in other words, hit rates are neither 0 nor 100\%). Also, the cases with a tighter activity threshold of $1 \mu \mathrm{M}$ are on average slightly better predicted than their counterparts using $10 \mu \mathrm{M}$. Specific cases with high- and low-precision performance will be discussed in Section Representative Case Studies. On the other hand, the sets with random molecules obtained much better results than those with approved drugs. Thus, if we order the four cases by average precision (dashed blue line in Figure 4), this gives the following performance hierarchy $\mathrm{D}>\mathrm{B}>\mathrm{C}>\mathrm{A}$ (i.e., $\mathrm{D}$ obtains higher average precision than $\mathrm{B}, \mathrm{B}$ better than $\mathrm{C}$, and $\mathrm{C}$ better than $\mathrm{A}$ ). Interestingly, this is the opposite ranking for the number of known targets $(\mathrm{A}>\mathrm{C}>\mathrm{B}>\mathrm{D})$. In other words, those sets with a higher number of known targets tend to be harder to predict.

However, the cause of obtaining lower predictive accuracy with approved drugs is not their higher number of known targets per se, but an underlying factor correlated with it: the query drug and its top hits, which should include some of the chemical derivatives that eventually led to this drug, often have a lower overlap in terms of known targets. One contributing factor for a low overlap is that two similar chemical structures do not always have affinity for the same targets. There is abundant literature analysing these pathological cases known as activity cliffs (Medina-Franco, 2013). Furthermore, even the top hits might not be highly similar to the query molecule, although this issue will become less frequent as more molecules are included in chemogenomics databases. Another contributing factor is that some of the top hits could have been tested against a range of targets in other studies, which might not have included the drug and thus this molecule would not have been tested against the targets (a lower precision for this query molecule would be 

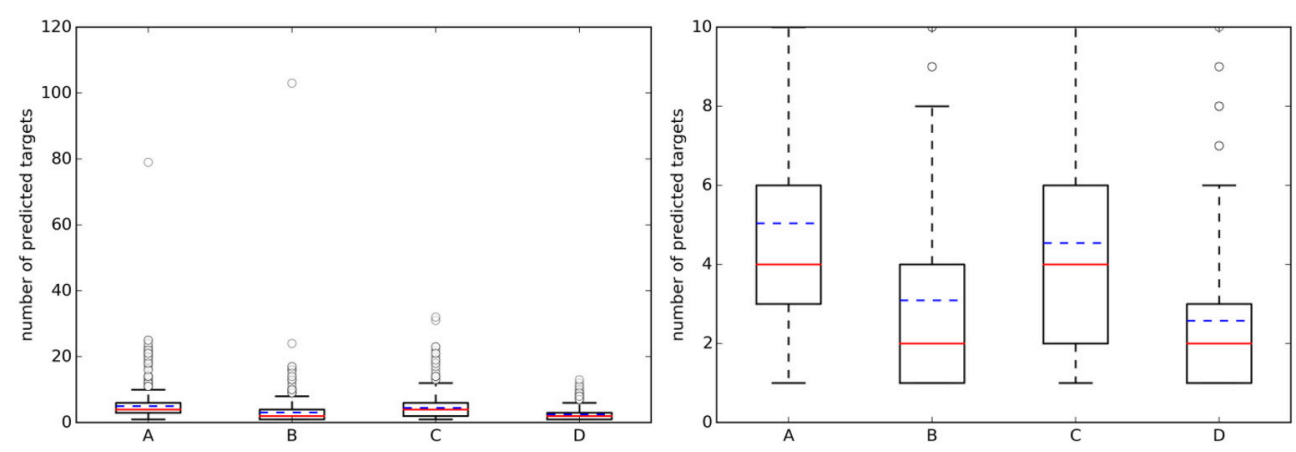

FIGURE 3 | (Left) Boxplots with the number of predicted targets (NPT) across query molecules using $k=5$. (Right) A zoom of the same boxplots on the right (the average number of known targets is marked with a dashed blue line, whereas the median is given by the continuous red line). $\mathrm{A}$, Approved, $10 \mu \mathrm{M}$; $\mathrm{B}$, Random, 10 $\mu \mathrm{M}$; C, Approved, $1 \mu \mathrm{M}$; D, Random, $1 \mu \mathrm{M}$.

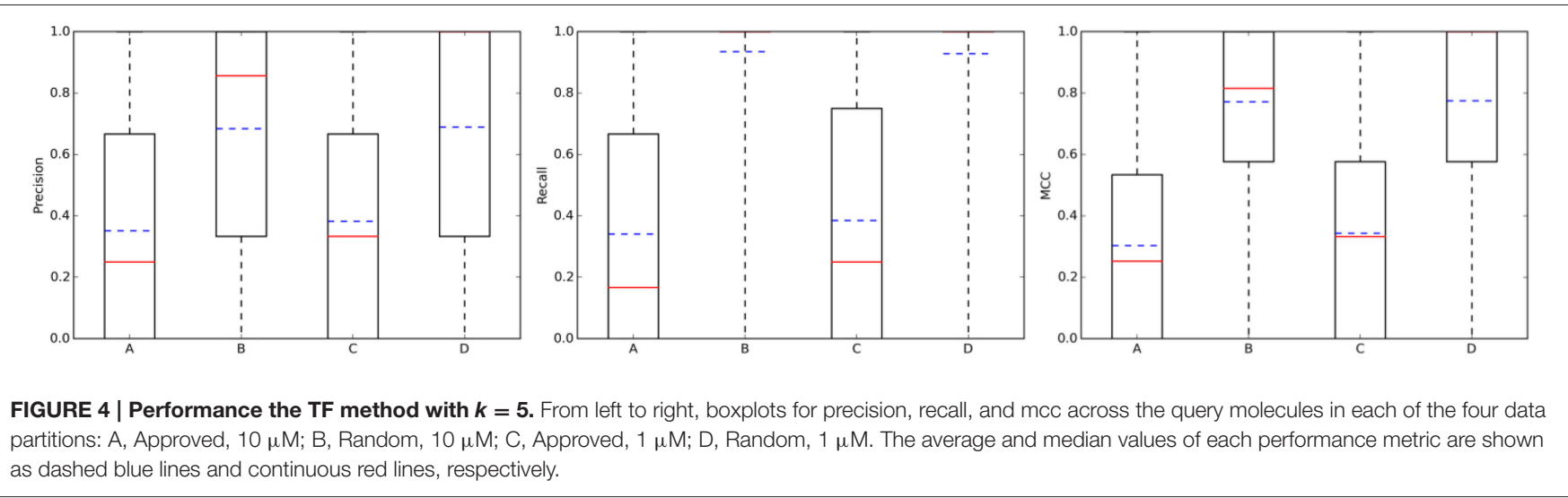

consequently obtained, as such targets would be perceived as false positives). Importantly, while these are not known targets of the drug, some are expected to become a known target once tested. In contrast, a molecule from the randomly-chosen set often has a larger overlap with its top hits (e.g., in set D, the predicted targets of 324 randomly-chosen molecules have precision $=1$, whereas those for just 35 randomly-chosen molecules have precision = 0 ). The latter cases are likely to arise from a situation where a chemical series is investigated against a set of related targets to be later abandoned (Waring et al., 2015). This would explain the lower number of known targets and the smaller predictive errors for these sets.

\section{How Many Known Targets of the Query Molecule Are Typically Missed?}

Addressing this question is necessary to estimate how many discoveries are being missed by the ligand-centric method, but it has not been investigated with regards to employed query molecule. Figure 4 presents the results in terms of recall (middle plot). Looking at the recall boxplots, only about $10 \%$ of the targets are on average missed in the sets of random molecules (i.e., recall $\sim 0.9$ ), whereas the mean of missed targets for approved drugs is about $65 \%$. A large part of these missed targets might be due to more intense research on the drug after approval than on its chemical derivatives, leading to many targets being tested in the former but not the latter.

On the other hand, the MCC boxplots (Figure 4 right plot) show the distribution of the total error across query molecules, with a high MCC necessarily meaning that the query molecule obtains low levels of both Type I and II errors. The latter occurs to most random molecules regardless of the activity threshold (almost $75 \%$ of these query molecules have MCCs higher than 0.6). In contrast, only a small proportion of approved drugs are in this category. Again, the performance hierarchy is $\mathrm{D}>\mathrm{B}>\mathrm{C}>\mathrm{A}$ for both recall and MCC. Here, a higher number of known targets in the query molecules is also correlated with the difficulty of predicting their targets, but this is also explained by the different ways in which the query molecules and their hits were tested against targets.

\section{Representative Case Studies}

Section How Many Targets Are Typically Hit by a Molecule? analyzed the NKT across query molecules. As discussed in Section How Many Predicted Targets Have to be Tested to Find a True Target?, the NKT of a molecule depends on its intrinsic polypharmacology, but also on how comprehensively the molecule has been tested across targets by the relevant scientific communities (we will call observed polypharmacology 
to the combination of these two factors). In the adopted TF method, the NPT of a molecule is given by the NKTs from its top 5 hits according to the dice score on MACCS fingerprints. Thus, the NPT for the query molecule depends in turn on the observed polypharmacology of each of these hits. In this section, we analyse two approved drugs representing cases where the difference in observed polypharmacology between the drug and its top hits are large in one direction $(\mathrm{NKT}>>\mathrm{NPT})$ or the other $(\mathrm{NKT}<<\mathrm{NPT})$.

The first case has nilotinib as the query molecule. Nilotinib was presented as a small-molecule selective tyrosine kinase inhibitor (Manley et al., 2010). However, we now know that this marketed drug has at least $\mathrm{NKT}=67$ known molecular targets under $10 \mu \mathrm{M}$, of which 14 are not kinases. In contrast, its top 5 hits collectively hit just nine targets $(\mathrm{NPT}=9)$. This is not surprising given the intense research interest in nilotinib as a targeted drug for the treatment of imatinib-resistant Chronic Myeloid Leukemia (Breccia and Alimena, 2010), but less so in its top hits from database molecules containing no drugs by construction. Figure $\mathbf{5 A}$ illustrates the proposed validation approach on nilotinib as the degree of overlap between the target spaces spanned by the query molecule and its top hits. Since predicted targets can only be either a true target or not, TP + $\mathrm{FP}=\mathrm{NPT}$. Likewise, known targets are either correctly predicted or not and thus TP $+\mathrm{FN}=\mathrm{NKT}$. As explained in Section Measuring Predictive Performance, the hit rate is given precision $=\mathrm{TP} / \mathrm{NPT}$, thus precision $=0.89$. In other words, the TF method retrospectively obtains an $89 \%$ hit rate for nilotinib (i.e., finding eight true targets of nilotinib in nine predicted targets). However, 59 known targets of nilotinib are missed by this method, as indicated by a low recall $=\mathrm{TP} / \mathrm{NKT}$ of 0.12 . This evidences that a method offering a high hit rate, while highly satisfying from a cost-effectiveness perspective, must be complemented by a high recall to be optimal.

Figure 5B shows the validation for the second case, which analyses the antimalarial agent chloroquine. $\mathrm{NKT}=3, \mathrm{NPT}=$ 21 , precision $=0.05$ and recall $=0.33$ are obtained in these case. This represents a modest hit rate of just 5\%, implying that a high experimental effort would have been associated to this discovery. However, the method obtains a higher recall with chloroquine than with nilotinib, which means that a lower proportion of known targets are being missed.

There are a total of $21 \mathrm{FP}$ target predictions in both query molecules. However, none of these target-ligand pairs have actually been tested (i.e., no bioactivity associated to them in ChEMBL). Since they come from the most similar molecules to the query, it is likely that some of these predictions will result in the discovery of new targets of these query molecules once tested. For instance, the only FP of nilotinib is human GRM5 (metabotropic glutamate receptor 5; CHEMBL3227), which is a known target of the 4th and 5th most similar database molecules to nilotinib (CHEMBL2346729 and CHEMBL2346732, both with submicromolar affinity for this target). Another exciting prospect is one of the 20 unconfirmed FPs from chloroquine, human CCR4 (C-C chemokine receptor type 4; CHEMBL2414), which also a clinically-relevant target and also been predicted by two of the top 5 hits (CHEMBL194930 and CHEMBL195203, both with single-digit micromolar potency).

\section{CONCLUSIONS}

We have shown that ligand-centric techniques for TF are capable of considering up to thousands of targets more than target-centric techniques. This important advantage means that ligand-centric techniques have their niche in TF. We have also discussed the limitations of current benchmarks to test TF methods and consequently we have designed a new benchmark that overcomes them. Using the proposed benchmark, it has been possible to investigate how reliable are ligand-centric methods for TF depending on the employed query molecule. Despite the simplicity of the adopted method and owing to the wealth of data on which it relies, we have found that only five predicted targets will have to be tested in order to find two true targets with potency better than $1 \mu \mathrm{M}$ on average over marketed drugs. This level of performance is already useful for prospective applications and it is encouraging that there is plenty of scope for methodological improvement. The latter will be particularly needed to reduce the high number of false negatives, i.e., known targets that are currently missed by ligandcentric techniques. It is worth noting that, while this issue
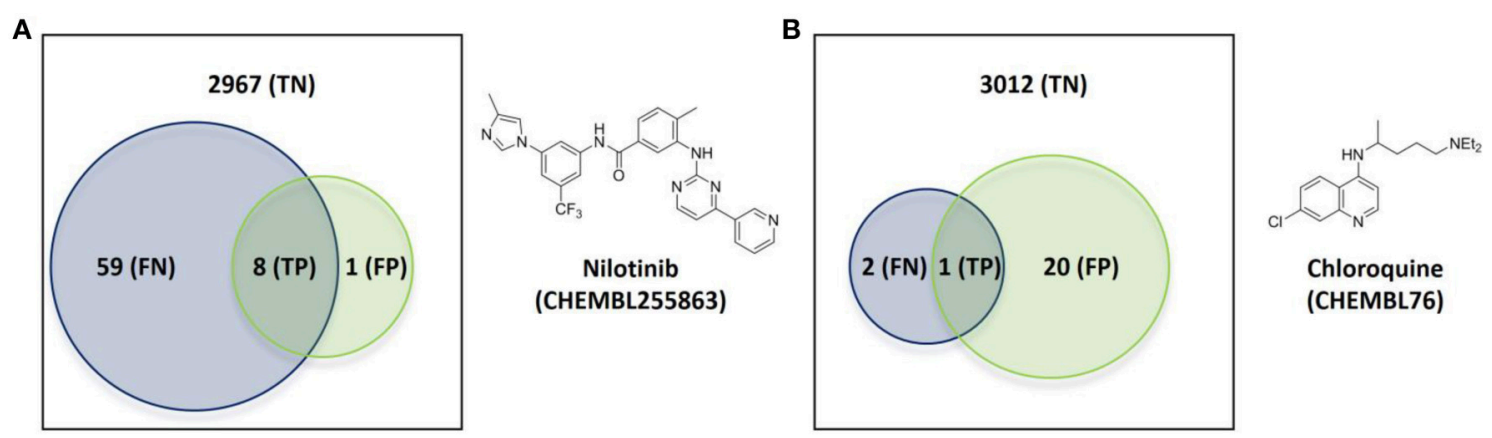

FIGURE 5 | Overlap of the target spaces under $10 \mu \mathrm{M}$ spanned by the query molecule (blue circle representing its known targets) and its top hits (green circle representing predicted targets given by the known targets of its top $\mathbf{5}$ hits) according to the employed TF method. (A) Nilotinib as the query molecule. (B) Chloroquine as the query molecule. 
has not been investigated yet for target-centric techniques, the many targets not considered by this class of techniques are by construction false negatives of any molecule that hits them. We have argued that this drawback is hard to appreciate as target-centric techniques only report predictive performance achieved on the typically much smaller set of considered targets.

The results for the set of randomly-selected molecules used as a control group are substantially better than those for approved drugs. We have discussed how the different way in which targets are tested against the query molecules and their top hits is the primary reason for this marked difference. Since approved drugs have been presumably tested against many more targets, we consider that their performance level is more realistic than that of the control set. Interestingly, we have identified an average of eight known targets under $10 \mu \mathrm{M}$ in approved drugs, which reinforces the notion that polypharmacology is a common and strong event. Lastly, high performance variability across query molecules has been observed in all cases. Thus, a promising avenue for future research consists in investigating which features make the target-ligand pair more

\section{REFERENCES}

AbdulHameed, M. D. M., Chaudhury, S., Singh, N., Sun, H., Wallqvist, A., and Tawa, G. J. (2012). Exploring polypharmacology using a ROCS-based target fishing approach. J. Chem. Inf. Model. 52, 492-505. doi: 10.1021/ci20 03544

Ain, Q. U., Aleksandrova, A., Roessler, F. D., and Ballester, P. J. (2015). Machine-learning scoring functions to improve structure-based binding affinity prediction and virtual screening. Wiley Interdiscip. Rev. Comput. Mol. Sci. 5, 405-424. doi: 10.1002/wcms.1225

Armstrong, M., Morris, G., Finn, P., Sharma, R., Moretti, L., Cooper, R., et al. (2010). ElectroShape: fast molecular similarity calculations incorporating shape, chirality and electrostatics. J. Comput. Aided Mol. Des. 24, 789-801. doi: 10.1007/s10822-010-9374-0

Ballester, P. J. (2011). Ultrafast shape recognition: method and applications. Future Med. Chem. 3, 65-78. doi: 10.4155/fmc. 10.280

Ballester, P. J., and Richards, W. G. (2007). Ultrafast shape recognition for similarity search in molecular databases. Proc. R. Soc. A Math. Phys. Eng. Sci. 463, 1307-1321. doi: 10.1098/rspa.2007.1823

Bento, A. P., Gaulton, A., Hersey, A., Bellis, L. J., Chambers, J., Davies, M., et al. (2014). The ChEMBL bioactivity database: an update. Nucleic Acids Res. 42, D1083-D1090. doi: 10.1093/nar/gkt1031

Breccia, M., and Alimena, G. (2010). Nilotinib: a second-generation tyrosine kinase inhibitor for chronic myeloid leukemia. Leuk. Res. 34, 129-134. doi: 10.1016/j.leukres.2009.08.031

Cereto-Massagué, A., Ojeda, M. J., Valls, C., Mulero, M., Pujadas, G., and GarciaVallve, S. (2015). Tools for in silico target fishing. Methods 71, 98-103. doi: 10.1016/j.ymeth.2014.09.006

Cheng, T., Pan, Y., Hao, M., Wang, Y., and Bryant, S. H. (2014). PubChem applications in drug discovery: a bibliometric analysis. Drug Discov. Today 19, 1751-1756. doi: 10.1016/j.drudis.2014.08.008

Cortés-Cabrera, A., Morris, G. M., Finn, P. W., Morreale, A., and Gago, F. (2013). Comparison of ultra-fast 2D and 3D ligand and target descriptors for side effect prediction and network analysis in polypharmacology. Br. J. Pharmacol. 170, 557-567. doi: 10.1111/bph.12294

Durant, J. L., Leland, B. A., Henry, D. R., and Nourse, J. G. (2002). Reoptimization of MDL keys for use in drug discovery. J. Chem. Inf. Model. 42, 1273-1280. doi: $10.1021 / \mathrm{ci0} 10132 \mathrm{r}$

Füllbeck, M., Dunkel, M., Hossbach, J., Daniel, P. T., and Preissner, R. (2009). Cellular fingerprints: a novel approach using large-scale cancer cell line data difficult to predict in order to assign a confidence score to each prediction.

Finally, we are releasing stand-along files implementing this benchmark, which permits easily comparing multiple TF methods on the same data. These files can also be used to serve as a template for the development of advanced ligand-centric TF methods, inspect our results or reproduce them from scratch.

\section{AUTHOR CONTRIBUTIONS}

PB conceived the study and wrote the manuscript. AP implemented the software and carried out the numerical experiments with the assistance of $\mathrm{CD}$. All authors reviewed the manuscript.

\section{ACKNOWLEDGMENTS}

This work has been carried out thanks to the support of the $\mathrm{A}^{*}$ MIDEX grant ( ${ }^{\circ}$ ANR-11-IDEX-0001-02) funded by the French Government «Investissements d'Avenir» program awarded to PB. for the identification of potential anticancer agents. Chem. Biol. Drug Des. 74, 439-448. doi: 10.1111/j.1747-0285.2009.00883.x

Gao, Z., Li, H., Zhang, H., Liu, X., Kang, L., Luo, X., et al. (2008). PDTD: a webaccessible protein database for drug target identification. BMC Bioinformatics 9:104. doi: 10.1186/1471-2105-9-104

Gfeller, D., Grosdidier, A., Wirth, M., Daina, A., Michielin, O., and Zoete, V. (2014). SwissTargetPrediction: a web server for target prediction of bioactive small molecules. Nucleic Acids Res. 42, W32-W38. Doi: 10.1093/nar/gku293

Holbeck, S. L., Collins, J. M., and Doroshow, J. H. (2010). Analysis of FDAapproved anti-cancer agents in the NCI60 panel of human tumor cell lines. Mol. Cancer Ther. 9, 1451-1460. doi: 10.1158/1535-7163.MCT-10-0106

Huang, H., Zhang, P., Qu, X. A., Sanseau, P., and Yang, L. (2014). Systematic prediction of drug combinations based on clinical side-effects. Sci. Rep. 4:7160. doi: $10.1038 /$ srep07160

Keiser, M. J., Roth, B. L., Armbruster, B. N., Ernsberger, P., Irwin, J. J., and Shoichet, B. K. (2007). Relating protein pharmacology by ligand chemistry. Nat. Biotechnol. 25, 197-206. doi: 10.1038/nbt1284

Keiser, M. J., Setola, V., Irwin, J. J., Laggner, C., Abbas, A. I., Hufeisen, S. J., et al. (2009). Predicting new molecular targets for known drugs. Nature 462, 175-181. doi: 10.1038/nature08506

Koutsoukas, A., Lowe, R., Kalantarmotamedi, Y., Mussa, H. Y., Klaffke, W., Mitchell, J. B. O., et al. (2013). In silico target predictions: defining a benchmarking data set and comparison of performance of the multiclass Naïve Bayes and Parzen-Rosenblatt window. J. Chem. Inf. Model. 53, 1957-1966. doi: $10.1021 / \mathrm{ci} 300435 \mathrm{j}$

Lamdrum, G. RDKit: Open-Source Cheminformatics. Available online at: http://www.rdkit.org/ (Accessed April 3, 2015).

Lavecchia, A., and Cerchia, C. (2015). In silico methods to address polypharmacology: current status, applications and future perspectives. Drug Discov. Today 21, 288-298. doi: 10.1016/j.drudis.2015.12.007

Lee, J., and Bogyo, M. (2013). Target deconvolution techniques in modern phenotypic profiling. Curr. Opin. Chem. Biol. 17, 118-126. doi: 10.1016/j.cbpa.2012.12.022

Liu, X., Xu, Y., Li, S., Wang, Y., Peng, J., Luo, C., et al. (2014). In Silico target fishing: addressing a "Big Data" problem by ligand-based similarity rankings with data fusion. J. Cheminform. 6:33. doi: 10.1186/1758-2946-6-33

Manley, P. W., Stiefl, N., Cowan-Jacob, S. W., Kaufman, S., Mestan, J., Wartmann, M., et al. (2010). Structural resemblances and comparisons of the relative pharmacological properties of imatinib and nilotinib. Bioorg. Med. Chem. 18, 6977-6986. doi: 10.1016/j.bmc.2010.08.026 
Martínez-Jiménez, F., Papadatos, G., Yang, L., Wallace, I. M., Kumar, V., Pieper, U., et al. (2013). Target prediction for an open access set of compounds active against Mycobacterium tuberculosis. PLoS Comput. Biol. 9:e1003253. doi: 10.1371/journal.pcbi.1003253

Medina-Franco, J. L. (2013). Activity cliffs: facts or artifacts? Chem. Biol. Drug Des. 81, 553-556. doi: 10.1111/cbdd.12115

Mestres, J., Gregori-Puigjané, E., Valverde, S., and Solé, R. V (2009). The topology of drug-target interaction networks: implicit dependence on drug properties and target families. Mol. Biosyst. 5, 1051-1057. doi: 10.1039/b90 $5821 \mathrm{~b}$

Mugumbate, G., Abrahams, K. A., Cox, J. A. G., Papadatos, G., van Westen, G., Lelièvre, J., et al. (2015). Mycobacterial dihydrofolate reductase inhibitors identified using chemogenomic methods and in vitro validation. PLoS ONE 10:e121492. doi: 10.1371/journal.pone.0121492

Nettles, J. H., Jenkins, J. L., Bender, A., Deng, Z., Davies, J. W., and Glick, M. (2006). Bridging chemical and biological space: "target fishing" using $2 \mathrm{D}$ and 3D molecular descriptors. J. Med. Chem. 49, 6802-6810. doi: 10.1021/jm0 60902w

Nigsch, F., Bender, A., Jenkins, J. L., and Mitchell, J. B. O. (2008). Ligand-target prediction using Winnow and naive Bayesian algorithms and the implications of overall performance statistics. J. Chem. Inf. Model. 48, 2313-2325. doi: $10.1021 / \mathrm{ci} 800079 \mathrm{x}$

Papadatos, G., Davies, M., Dedman, N., Chambers, J., Gaulton, A., Siddle, J., et al. (2016). SureChEMBL: a large-scale, chemically annotated patent document database. Nucleic Acids Res. 44, D1220-D1228. doi: 10.1093/nar/gkv1253

Rogers, D., and Hahn, M. (2010). Extended-connectivity fingerprints. J. Chem. Inf. Model. 50, 742-754. doi: 10.1021/ci100050t

Schneider, G. (2010). Virtual screening: an endless staircase? Nat. Rev. Drug Discov. 9, 273-276. doi: 10.1038/nrd3139

Schomburg, K. T., and Rarey, M. (2014). Benchmark data sets for structurebased computational target prediction. J. Chem. Inf. Model. 54, 2261-2274. doi: $10.1021 /$ ci500131x

Speck-Planche, A., and Cordeiro, M. N. D. S. (2015). Multi-Target QSAR approaches for modeling protein inhibitors. Simultaneous prediction of activities against biomacromolecules present in gram-negative bacteria. Curr. Top. Med. Chem. 15, 1801-1813. doi: 10.2174/1568026615666150506144814
Sukumar, N., and Das, S. (2011). Current trends in virtual high throughput screening using ligand-based and structure-based methods. Comb. Chem. High Throughput Screen. 14, 872-888. doi: 10.2174/138620711797537120

Ursu, A., and Waldmann, H. (2015). Hide and seek: identification and confirmation of small molecule protein targets. Bioorg. Med. Chem. Lett. 25, 3079-3086. doi: 10.1016/j.bmcl.2015.06.023

van Laarhoven, T., Nabuurs, S. B., and Marchiori, E. (2011). Gaussian interaction profile kernels for predicting drug-target interaction. Bioinformatics 27, 3036-3043. doi: 10.1093/bioinformatics/btr500

Wang, L., Ma, C., Wipf, P., Liu, H., Su, W., and Xie, X.-Q. (2013). TargetHunter: an in silico target identification tool for predicting therapeutic potential of small organic molecules based on chemogenomic database. AAPS J. 15, 395-406. doi: 10.1208/s12248-012-9449-z

Waring, M. J., Arrowsmith, J., Leach, A. R., Leeson, P. D., Mandrell, S., Owen, R. M., et al. (2015). An analysis of the attrition of drug candidates from four major pharmaceutical companies. Nat. Rev. Drug Discov. 14, 475-486. doi: $10.1038 / \mathrm{nrd} 4609$

Willett, P. (2014). The calculation of molecular structural similarity: principles and practice. Mol. Inform. 33, 403-413. doi: 10.1002/minf.201400024

Yu, H., Chen, J., Xu, X., Li, Y., Zhao, H., Fang, Y., et al. (2012). A systematic prediction of multiple drug-target interactions from chemical, genomic, and pharmacological data. PLoS ONE 7:e37608. doi: 10.1371/journal.pone.0037608

Zanni, R., Gálvez-Llompart, M., Gálvez, J., and García-Domenech, R. (2014) QSAR multi-target in drug discovery: a review. Curr. Comput. Aided. Drug Des. 10, 129-136. doi: 10.2174/157340991002140708105124

Conflict of Interest Statement: The authors declare that the research was conducted in the absence of any commercial or financial relationships that could be construed as a potential conflict of interest.

Copyright (C) 2016 Peón, Dang and Ballester. This is an open-access article distributed under the terms of the Creative Commons Attribution License (CC BY). The use, distribution or reproduction in other forums is permitted, provided the original author(s) or licensor are credited and that the original publication in this journal is cited, in accordance with accepted academic practice. No use, distribution or reproduction is permitted which does not comply with these terms. 